Proceedings of the International School and Conference on Optics and Optical Materials, ISCOM07, Belgrade, Serbia, September 3-7, 2007

\title{
Using 2D Distributed Feedback in Optical Laser
}

\author{
V.R. Baryshev*, N.S. Ginzburg, A.M. Malkin
}

AND A.S. SERGEEV

Institute of Applied Physics, RAS, Nizhny Novgorod, Russia

Dynamics of a laser exploiting two-dimensional distributed feedback is considered. Two-dimensional distributed feedback can be realised using a dielectric structure (two-dimensional Bragg resonator) with the width having double-periodical modulation. It is shown that such a structure can provide production of spatial coherent radiation from extended active medium of planar geometry.

PACS numbers: 42.55.Tr, 42.60.Da

\section{Introduction}

The concept of traditional distributed feedback (DFD) laser is based on the propagation of guided waves in a dielectric structure with single periodical modulation $[1,2]$. Such structure forms 1D Bragg resonator where two counterpropagating waves provide a feedback circle. The selective properties of the above resonator are sufficient for mode control over the longitudinal index. In planar geometry spatial synchronization of radiation over another transverse coordinate can be provided by diffraction if the transverse size of the system $b$ is restricted by the Fresnel condition $b^{2} / l \lambda<1$, where $l$ is the system length. In our paper we describe an effective method for the spatial synchronization of radiation in the case of large Fresnel parameter: $b^{2} / l \lambda \gg 1$ that is based on using $2 \mathrm{D}$ distributed feedback. In microwave electronics $2 \mathrm{D}$ distributed feedback was suggested in $[3,4]$ to produce spatially coherent radiation from either sheet or annular high-current relativistic electron beam with the transverse size greatly exceeding the wavelength and can be realised in planar and coaxial 2D Bragg cavities with a double-periodical corrugation of the metallic walls. In this structure mutual scattering of the electromagnetic energy fluxes propagating in the forward, backward and transverse directions (relative to the direction of the electron beam propagation) takes place. The additional transverse electromagnetic energy fluxes act to synchronise radiation from different parts of a large size electron beam.

*corresponding author; e-mail: baryshev@appl.sci-nnov.ru 
A laser active medium should provide an isotropic amplification of all the partial waves independently of directions of their propagation. In optics the 2D distributed feedback can be realised using a dielectric structure with the width having double-periodical sinusoidal modulation

$$
b=\frac{b_{0}}{2}[\cos (\bar{h}(x-z))+\cos (\bar{h}(x+z))],
$$

where $b_{0}$ is the amplitude of corrugation, $\bar{h}=2 \pi / D, D$ is the period of corrugation along $x$ and $z$ directions. Under Bragg resonance conditions: $h=\bar{h}$ ( $h$ is the wave

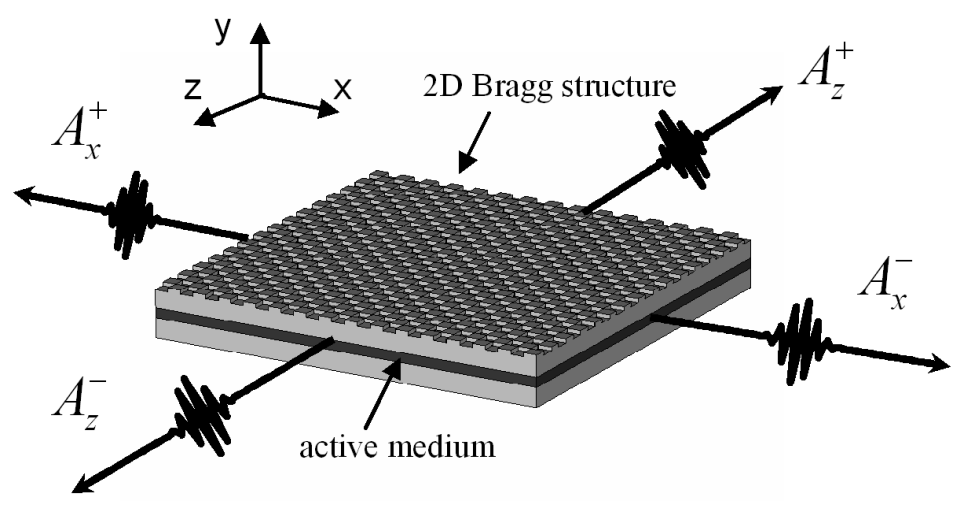

Fig. 1. 2 D DFB laser schematic.

number of the fundamental eigenmode of the regular dielectric waveguide) we can arrange coupling and mutual scattering of four wave fluxes propagating in the $\pm z$ and $\pm x$ directions (see Fig. 1):

$$
\boldsymbol{E}=\boldsymbol{E}_{0}(y) \operatorname{Re}\left(\left(A_{-}^{z} \mathrm{e}^{-\mathrm{i} h z}+A_{+}^{z} \mathrm{e}^{\mathrm{i} h z}+A_{-}^{x} \mathrm{e}^{-\mathrm{i} h x}+A_{+}^{x} \mathrm{e}^{\mathrm{i} h x}\right) \mathrm{e}^{\mathrm{i} \omega t}\right),
$$

where $\boldsymbol{E}_{0}(y)$ is the transverse structure of the fundamental eigenmode of the dielectric waveguide without the corrugation. For practical applications similarly to the case of metallic waveguide [4] it is sufficient to approximate sinusoidal modulation by chessboard function (see Fig. 1).

\section{The model}

We will describe the interaction between the field and the active medium within the framework of semiclassical approach [1, 5]. Taking into account the field presentation (3) we present the polarization and the population inversion in the form [5]:

$$
\begin{aligned}
& P=\operatorname{Re}\left(\mathrm{i}\left(P_{z}^{+} \mathrm{e}^{\mathrm{i} \bar{h} z}+P_{z}^{-} \mathrm{e}^{-\mathrm{i} \bar{h} z}+P_{x}^{+} \mathrm{e}^{\mathrm{i} \bar{h} x}+P_{x}^{-} \mathrm{e}^{-\mathrm{i} \bar{h} x}\right) \mathrm{e}^{\mathrm{i} \omega_{0} t}\right) \\
& \rho=\rho_{0}+\operatorname{Re}\left(\rho_{2 z} \mathrm{e}^{2 \mathrm{i} \bar{h} z}+\rho_{2 x} \mathrm{e}^{2 \mathrm{i} \bar{h} x}+\rho_{z-x} \mathrm{e}^{2 \mathrm{i} \bar{h}(z-x)}+\rho_{z+x} \mathrm{e}^{2 \mathrm{i} \bar{h}(z+x)}\right),
\end{aligned}
$$

where $P_{x, z}^{ \pm}, \rho_{0}, \rho_{2 z}, \rho_{2 x}, \rho_{z-x}, \rho_{z+x}$, are the slowly varying amplitudes. 
The process of amplification of these waves in the active medium and their mutual scattering on the Bragg corrugation and nonlinear corrugation emerging from the modulation of inversion can be described by the averaged equations

$$
\begin{aligned}
& \left(\mp \frac{\partial}{\partial Z}+\frac{\partial}{\partial \tau}\right) \hat{A}_{z}^{ \pm}+\mathrm{i}\left(\hat{A}_{x}^{+}+\hat{A}_{x}^{-}\right)=\hat{P}_{z}^{ \pm}, \\
& \left(\mp \frac{\partial}{\partial X}+\frac{\partial}{\partial \tau}\right) \hat{A}_{x}^{ \pm}+\mathrm{i}\left(\hat{A}_{z}^{+}+\hat{A}_{z}^{-}\right)=\hat{P}_{x}^{ \pm}, \\
& \frac{\partial \hat{\rho}_{0}}{\partial \tau}+\frac{\hat{\rho}_{0}-1}{\hat{T}_{1}}=-\left(\hat{A}_{z}^{+} \hat{P}_{z}^{+*}+\hat{A}_{z}^{-} \hat{P}_{z}^{-*}+\hat{A}_{x}^{+} \hat{P}_{x}^{+*}+\hat{A}_{x}^{-} \hat{P}_{x}^{-*}\right) \\
& \frac{\partial \hat{\rho}_{2 z}}{\partial \tau}+\frac{\hat{\rho}_{2 z}}{\hat{T}_{1}}=-\left(\hat{A}_{z}^{+} \hat{P}_{z}^{-*}+\hat{A}_{z}^{-} \hat{P}_{z}^{+*}\right) \\
& \frac{\partial \hat{\rho}_{2 x}}{\partial \tau}+\frac{\hat{\rho}_{2 x}}{\hat{T}_{1}}=-\left(\hat{A}_{x}^{+} \hat{P}_{x}^{-*}+\hat{A}_{x}^{-} \hat{P}_{x}^{+*}\right) \\
& \frac{\partial \hat{\rho}_{z+x}}{\partial \tau}+\frac{\hat{\rho}_{z+x}}{\hat{T}_{1}}=-\left(\hat{A}_{z}^{+} \hat{P}_{x}^{-*}+\hat{A}_{z}^{-*} \hat{P}_{x}^{+}+\hat{A}_{x}^{+} \hat{P}_{z}^{-*}+\hat{A}_{x}^{-*} \hat{P}_{z}^{+}\right), \\
& \frac{\partial \hat{\rho}_{z-x}}{\partial \tau}+\frac{\hat{\rho}_{z-x}}{\hat{T}_{1}}=-\left(\hat{A}_{z}^{+} \hat{P}_{x}^{+*}+\hat{A}_{z}^{-*} \hat{P}_{x}^{-}+\hat{A}_{x}^{+*} \hat{P}_{z}^{+}+\hat{A}_{x}^{-} \hat{P}_{z}^{-*}\right),
\end{aligned}
$$

where $\rho_{\mathrm{e}}$ is an equilibrium value of population inversion, $\mu$ is dipole moment, $T_{1}$ and $T_{2}$ are the relaxation times, $\omega_{0}$ is the transition frequency $\hat{\rho}=\rho / \rho_{\mathrm{e}}$, $\hat{P}_{x, z}^{ \pm}=P_{x, z}^{ \pm} \alpha\left(\rho_{\mathrm{e}} \hbar \omega_{0}^{3} / 2 \pi\right)^{-1 / 2}, \hat{A}_{x, z}^{ \pm}=A_{x, z}^{ \pm} \omega_{0}^{2}\left(\rho_{\mathrm{e}} \hbar \omega_{0}^{3} / 2 \pi\right)^{-1 / 2} / 2 \pi c, \hat{T}_{1,2}=\alpha \omega_{0} T_{1,2}$, $X=\alpha \omega_{0} x / c, Z=\alpha \omega_{0} z / c, \tau=\alpha \omega_{0} t$. Here $\alpha=b_{0} \omega_{0} \varepsilon \boldsymbol{E}_{0}^{2}(d / 2) / \pi h c \int_{-\infty}^{\infty} \boldsymbol{E}_{0}^{2}(y) \mathrm{d} y$ is the coupling coefficient of partial waves in the case of chessboard corrugation, $b_{0}$ is the corrugation amplitude, $d$ is the mean width of the dielectric layer, $\boldsymbol{E}_{0}(d / 2)$ is the unperturbed mode amplitude on the corrugation (see (2)), $\varepsilon$ is the dielectric constant. Obtaining (5) we used the balance approximation assuming that transverse relaxation time $T_{2}$ is small comparing to other time values, expressing for example $\hat{P}_{z}^{+}$components of polarization as

$$
\hat{P}_{z}^{+}=\beta \hat{T}_{2}\left(2 \hat{A}_{z}^{+} \hat{\rho}_{0}+\hat{A}_{z}^{-} \hat{\rho}_{2 z}+\hat{A}_{x}^{+} \hat{\rho}_{z-x}+\hat{A}_{x}^{-} \hat{\rho}_{z+x}\right)
$$

where $\beta=\pi \rho_{\mathrm{e}}|\mu|^{2} / \alpha^{2} \hbar \omega_{0}^{3}$ is normalized density of active atoms, $\hat{T}_{2}=\alpha \omega_{0} T_{2}$.

\section{Results and discussion}

According to $[3,4]$ the $2 \mathrm{D}$ Bragg resonator possesses high selectivity over both the longitudinal and the transverse indices. This selectivity originates from output of radiation not only in the longitudinal $\pm z$ directions (similar to $1 \mathrm{D}$ Bragg resonators), but additionally in the transverse $\pm x$ directions. Peculiarity of the $2 \mathrm{D}$ Bragg structures is the existence of the high- $Q$ eigenmodes inside the Bragg resonance zone without any defects of the periodicity. The highest $Q$-factor is achieved at the precise Bragg frequency. Self-excitation conditions of that fundamental mode can be put in a form: $4 \beta \hat{T}_{2} l_{z}^{2} l_{x} / \pi^{2}=1$.

Nonlinear stage of the oscillation was studied by means of numerical simulation of Eq. (5). Temporal dependences of total energy of electromagnetic field 


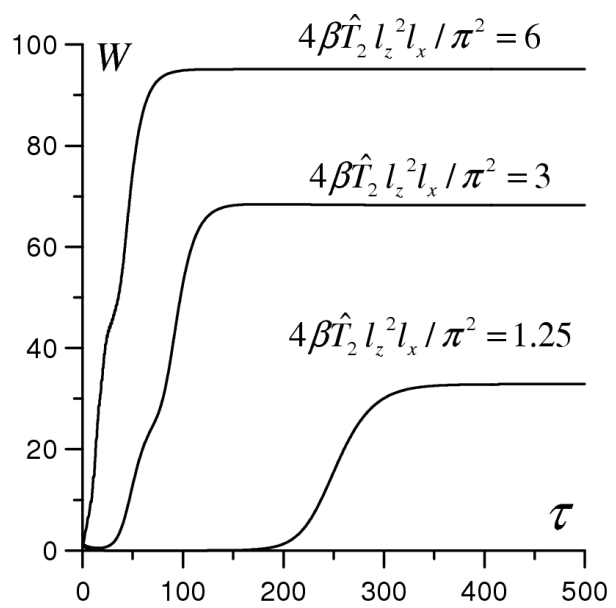

Fig. 2. Temporal dependences of total energy of electromagnetic field at different values of the excess over the threshold.

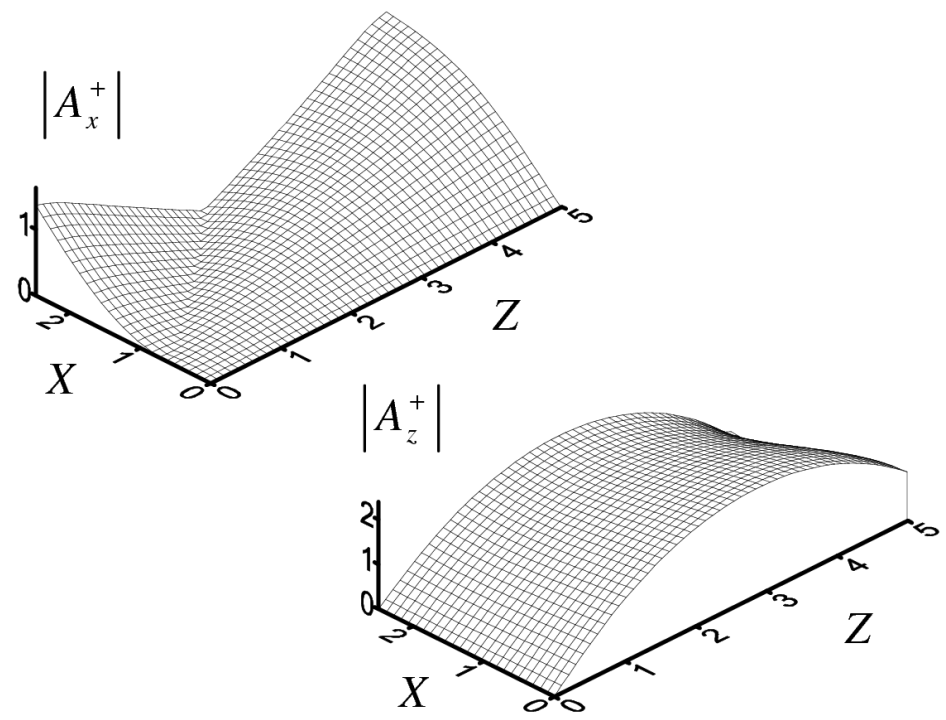

Fig. 3. The distributions of amplitudes of partial waves in the stationary regime of oscillations.

at different values of the excess over the threshold are presented in Fig. 2, which illustrates the process of establishment of stationary regime of oscillations. In Fig. 3 the distributions of amplitudes of partial waves in this regime are depicted at $L_{z}=5, L_{x}=2.5, \beta \hat{T}_{2}=0.1, \hat{T}_{1}=1$. Spectra of the signal on the initial (linear) stage of transient process and in the steady-state regime of oscillations are presented in Fig. 4. In this case besides the fundamental mode the threshold of self-excitation is exceeded for some other modes, so in the initial stage (Fig. 4a) 

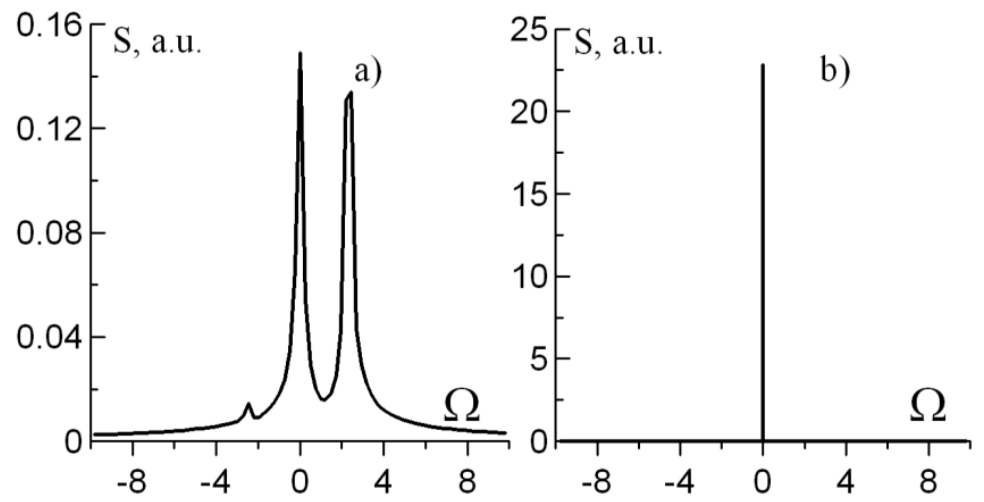

Fig. 4. Spectra of the signal on the initial (linear) stage of transient process (a) and in the steady-state regime of oscillations (b).

spectral maxima corresponding to the frequencies of these modes can be seen. However, nonlinear mode competition leads to the establishment of steady-state single-frequency (Fig. 4b) regime with a frequency and partial waves amplitudes distribution similar to those of the fundamental mode of the Bragg resonator (see $[1,2])$. Thus, simulation demonstrates the possibility of using the 2D Bragg structures for spatial synchronization of radiation of extended active medium of planar geometry with large Fresnel parameter.

\section{References}

[1] A. Yariv, Quantum Electronics, Wiley, New York 1975.

[2] H. Kogelnik, C.V. Shank, J. Appl. Phys. 43, 2327 (1972).

[3] N.S. Ginzburg, N.Yu. Peskov, A.S. Sergeev, Opt. Commun. 96, 254 (1993).

[4] N.S. Ginzburg, N.Yu. Peskov, A.S. Sergeev, Phys. Rev. E 60, 935 (1999).

[5] A.V. Andreev, Usp. Fiz. Nauk 160, 1 (1990). 